\title{
Poly(E-caprolactone) nanocapsules carrying the herbicide atrazine: effect of chitosan-coating agent on physico-chemical stability and herbicide release profile
}

\author{
R. Grillo • A. H. Rosa $・$ L. F. Fraceto
}

Received: 7 February 2013/Revised: 22 June 2013/Accepted: 8 August 2013/Published online: 29 August 2013

(C) Islamic Azad University (IAU) 2013

\begin{abstract}
Polymeric nanoparticles can be used as carrier systems for a variety of bioactive compounds, altering the physico-chemical properties of the substances that are incorporated in the particle matrix. Coating techniques are employed to modify the interactions of the particles with the target medium. The objective of this work was to prepare nanocapsules of poly( $\varepsilon$-caprolactone) containing the herbicide atrazine and study the effect of coating the nanocapsule surfaces using different concentrations of chitosan. The encapsulation efficiency, release kinetics profile, and physico-chemical stability of the formulations were evaluated. Encapsulation percentages of 64-84\% were achieved. The release kinetics profile of the herbicide was altered when the nanocapsules were coated, and the release mechanism could be described by a combination of diffusion and relaxation of the polymeric chains. The stability of the formulations was influenced by the concentration of chitosan used in the preparation procedure. Given the global application of herbicides in agriculture, the results indicate that their association with coated modified release carrier systems could be beneficial from both ecological and economic perspectives. These systems offer delivery that is more efficient, as well as improved adhesion of herbicides to the target plants.
\end{abstract}

R. Grillo · A. H. Rosa · L. F. Fraceto ( $₫)$

Department of Environmental Engineering, UNESP-Univ.

Estadual Paulista, Avenida Três de Março, no 511, Sorocaba,

SP 18087-180, Brazil

e-mail: leonardo@sorocaba.unesp.br

R. Grillo · L. F. Fraceto

Department of Biochemistry, Institute of Biology, UNICAMP, Cidade Universitária Zeferino Vaz, s/n, Campinas, SP, Brazil
Keywords Environmental chemistry · Atrazine · Coated polymeric nanoparticles $\cdot$ Chitosan

\section{Introduction}

Colloidal nanoparticle systems can be used as carriers of bioactive compounds, whose properties then become different to those obtained when the materials are prepared at the macroscopic scale (Kumar et al. 2012; Wu et al. 2011). In addition to particle size, classification of a system as nanometric can be based on a variety of other parameters, including particle structure, porosity, and surface area (Maynard 2011).

Many studies reported in the literature have focused on the association of bioactive compounds with nanoparticles as a means of steering molecules to specific target sites, protecting the active principal against degradation, and modifying its release profile (Radad et al. 2012; Kumar et al. 2012). Nanoparticles can be produced using biocompatible and biodegradable synthetic polymers such as poly(E-caprolactone) (PCL) (Dash and Konkimalla 2012), poly(glycolic acid) (PGA), poly(lactic acid) (PLA), and poly(lactic-coglycolide) (PLGA), as well as natural polymers including gelatin, chitosan (Garcia-Fuentes and Alonso 2012), alginate, and polysaccharides such as cellulose (Sabir et al. 2009).

Growth in the development of polymeric nanoparticles, and the desire to migrate this technology to new applications, has led to the need to modify particle surfaces in order to enhance the delivery of the bioactive compounds contained within the particles, and improve adhesion of nanoparticles to target substrates. One way of altering the nanoparticle surface is to coat it with oppositely charged polymers. Kumar et al. (2012) described a variety of 
coating techniques, involving emulsification, evaporation, coacervation, and nanoprecipitation. The coating of nanoparticles is an effective means of improving the mucoadhesion of bioactive compounds (Prego et al. 2005) delivered orally (Tobio et al. 2000; Mazzarino et al. 2012) and ocularly (Calvo et al. 1997; Nagarwal et al. 2012), increasing the efficiency of chemotherapeutic pharmaceuticals (Chakravarthi and Robinson 2011; Bilensoy et al. 2009), inducing humoral and cellular immunity against the influenza virus (Gupta et al. 2011), and increasing the stability and controlled release of peptides (Gref et al. 2001)

Mazzarino et al. (2012) prepared PCL nanoparticles coated with chitosan and containing the compound curcumin, and observed that the coated particles were stable and had a strong electrostatic interaction with the mucosa. Similar results were obtained by Calvo et al. (1997), who coated nanocapsules (NC) of poly(L-lysine) and poly( $\varepsilon$ caprolactone) with chitosan and associated them with the drug indometacin, in order to develop a new ocular release system. In this case, chitosan was used to increase the mucoadhesion of the particles, given that the epithelium of the cornea is negatively charged. The coating did not alter the release profile of the bioactive compound, but significantly increased the quantity of drug in the ocular region.

Use of the coating technique can induce different interactions between the nanoparticles and the target substrate, due to modification of the charge and other properties of the surface (Calvo et al. 1997; Mazzarino et al. 2012; Prego et al. 2005).

Most of the reported studies concerning the preparation of polymeric nanoparticles have focused on their use as carriers in medical applications (Grillo et al. 2010a; Clemente-Napimoga et al. 2012; Moraes et al. 2011; de Melo et al. 2011; Mora-Huertas et al. 2010). However, recent work has involved the application of polymeric nanoparticles in agriculture, including the development of formulations for the release of herbicides (Grillo et al. 2011, 2012; Silva et al. 2011; Grillo et al. 2010b; Silva et al. 2010, 2012). The practical use of nanotechnology in the field environment can bring a series of benefits, including improvements in the stability of formulations, alterations in the release of the active principle, protection against external agents (oxidation, light, hydrolysis, and evaporation), and improved delivery of the active principle to plants. Nanoparticles can provide better adhesion to the waxy surface of the leaf, favoring adsorption of the herbicide and maximizing biological activity (Taylor 2011), amongst other effects (Hack et al. 2012).

Recent work reported the preparation and characterization of formulations of PCL NC containing different triazine herbicides (Grillo et al. 2012). Association of the herbicides with the $\mathrm{NC}$ altered the release profiles of the active principles, and the formulations presented good colloidal stability, as well as reduced genotoxicity, due to the modified release of the herbicides. Amongst the various triazine herbicides available on the market, atrazine (ATZ) is one of the compounds most widely used globally in agriculture for the pre- and post-emergent control of invasive broadleaved and graminaceous plants (Breckenridge et al. 2010).

The objective of this work was to use previously prepared PCL NC containing ATZ (Grillo et al. 2012) to study the effect of coating the NC surface with different concentrations of chitosan. The physico-chemical stability of the NC was evaluated, and the release profiles of ATZ were investigated, in order to obtain information relevant to the formulation of chitosan-coated PCL NC that offered improved adhesion to target plants and that would be suitable for use in new agricultural applications.

\section{Materials and methods}

\section{Materials}

Atrazine, poly- $\varepsilon$-caprolactone (PCL, MW $80 \mathrm{kDa}$ ), Polysorbate 80 (Tween 80 , MW 1,310), sorbitan monostearate (Span 60), and chitosan (CS, low molecular weight) were purchased from Sigma-Aldrich. Oil consisting of the triglycerides of capric and caprylic acids (Myritol 318) was obtained from ChemSpecs (Brazil). The solvents used in the chromatographic analyses were HPLC grade acetonitrile (JT Baker) and Milli-Q water. The solutions were filtered using 0.22- $\mu \mathrm{m}$ nylon membranes (Millipore, Bedford, USA). All other reagents and solvents were sourced within Brazil.

Preparation of the polymeric nanocapsules containing atrazine

The nanocapsules coated with chitosan were prepared by the method of interfacial deposition of preformed polymer, with modifications (Grillo et al. 2012). This technique involves the mixing of an organic phase into an aqueous phase. The organic phase was composed of $100 \mathrm{mg}$ of polymer (PCL), $30 \mathrm{~mL}$ of organic solvent (acetone), $200 \mathrm{mg}$ of oil (Myritol 318), $40 \mathrm{mg}$ of Span 60, and $10 \mathrm{mg}$ of herbicide (ATZ). The aqueous phase consisted of $30 \mathrm{~mL}$ of a solution containing $60 \mathrm{mg}$ of Tween 80 . After dissolving the components of both phases, the organic phase was slowly inserted into the aqueous phase, with magnetic stirring. The resulting suspension was maintained under agitation for $10 \mathrm{~min}$, after which the organic solvent was evaporated under reduced pressure using a rotary evaporator (Fessi et al. 1989). The nanoparticle suspension was 
evaporated to a final volume of $10 \mathrm{~mL}$, resulting in an herbicide concentration of $1 \mathrm{mg} / \mathrm{mL}$. Different quantities of chitosan $(0,8,10,20,30$, and $40 \mu \mathrm{g} / \mathrm{mL})$ were then added, using a stock solution of $500 \mu \mathrm{g} / \mathrm{mL}$ chitosan prepared at $\mathrm{pH} \mathrm{4}$, and the mixtures were kept under agitation overnight. The resulting samples were stored in the dark at $25^{\circ} \mathrm{C}$ for later analysis.

\section{Measurement of encapsulation efficiency}

The total quantity $(100 \%)$ of herbicide present in the suspensions of polymeric NC coated with chitosan was determined by diluting the suspensions with acetonitrile. This solvent is able to completely dissolve the nanoparticle structure hence disrupting the system and dispersing the active component present within the matrix (Schaffazick et al. 2003).

After this step, the suspension was filtered using a 0.22$\mu \mathrm{m}$ membrane (Millipore), and the herbicide was quantified by high-performance liquid chromatography (HPLC, Varian ProStar), employing a Phenomenex column (Gemini $5 \mu \mathrm{C}_{18} 110 \mathrm{~A}, 250 \times 4.60 \mathrm{~mm}$ ), acetonitrile/water (50:50, v/v) eluent, a flow rate of $1.0 \mathrm{~mL} / \mathrm{min}$, and a detector wavelength of $225 \mathrm{~nm}$. The analytical methodology was previously validated according to the ICH guidelines (ICH 1996); the analytical curve presented a linear correlation coefficient $\left(r^{2}\right)$ of 0.999 , and the detection and quantification limits were 0.57 and $1.91 \mu \mathrm{g} / \mathrm{mL}$, respectively.

The quantity of herbicide encapsulated in the $\mathrm{NC}$ was determined by the ultrafiltration/centrifugation method. The coated $\mathrm{NC}$ were centrifuged using an ultrafiltration $(2,000 \times g)$ unit composed of $30 \mathrm{kDa}$ regenerated cellulose (Microcon, Millipore), after which the herbicide was quantified in the filtrate (corresponding to the amount of free herbicide). The encapsulation efficiency was determined as the difference between the total amount of herbicide present in the samples $(100 \%)$ and the amount that was not encapsulated.

\section{Evaluation of nanocapsule stability}

The stability of the coated NC formulations, with or without ATZ, was determined using several physicochemical parameters. Measurements were made of the hydrodynamic diameter, polydispersion index, zeta potential, and $\mathrm{pH}$, as a function of time (after $0,15,30,60$, and 90 days) (Guterres et al. 2010).

Determination of nanocapsule diameter and polydispersion index The light scattering technique was used to determine the average diameter and size distribution (polydispersion) of the NC. The procedure was performed by diluting the coated $\mathrm{NC}$ with deionized water $(1: 100, \mathrm{v} / \mathrm{v})$, prior to measurement at $25^{\circ} \mathrm{C}$ using a Malvern Instruments Zeta Plus particle analyzer with the detector at a fixed angle of $90^{\circ}$. Each result was expressed as the average of three determinations (Govender et al. 2000; Grillo et al. 2012).

\section{Determination of zeta potential}

The electrical potential at the hydrodynamic shearing plane of the NC was evaluated by determination of the zeta potential using the Malvern Instruments Zeta Plus analyzer. The analyses were performed after diluting the suspensions of nanoparticles coated with chitosan in deionized water $(1: 100, \mathrm{v} / \mathrm{v})$. The formulations presented conductivity values exceeding $0.25 \mu \mathrm{S} / \mathrm{cm}$. The results were expressed as the average of three determinations (Grillo et al. 2012).

\section{Measurement of polymer chemical stability}

The chemical stability of the polymer was evaluated using measurement of the $\mathrm{pH}$ of the suspension as a function of time, since alterations in this parameter can be indicative of polymer chemical degradation (Guterres et al. 2010; Schaffazick et al. 2003). The NC suspension $\mathrm{pH}$ was measured over a period of 90 days, using a $\mathrm{pH}$ meter (Tecnal) that had been previously calibrated with $\mathrm{pH} 7$ and pH 4 buffer solutions.

\section{In vitro release kinetics}

In vitro release kinetics experiments were used to investigate how different quantities of chitosan used to coat the PCL NC affected the release profile of ATZ. A two-compartment system was used, in which the donor and acceptor compartments were separated by a Spectrapore cellulose membrane with a molecular exclusion pore size of $1 \mathrm{kDa}$. The sample ( $2 \mathrm{~mL}$ of the formulation) was placed in the donor compartment, and the acceptor compartment was filled with solvent $(80 \mathrm{~mL}$ of water) maintained under gentle agitation (Grillo et al. 2012; Paavola et al. 1995). Samples were collected from the acceptor compartment for analysis by HPLC at $15 \mathrm{~min}$ intervals during the first hour, and then at intervals of $30 \mathrm{~min}$ and $1 \mathrm{~h}$, over a period of approximately 5 days, until the release stabilized. The peak area values were converted into the percentage of herbicide released as a function of time, and all measurements were performed in triplicate.

The release efficiency (RE) was calculated from the areas under the release profile curves obtained for the herbicide alone or for the herbicide in the suspensions of coated NC, obtained at time intervals $(\mathrm{t})$ between zero and 5.5 days, divided by the area under the upper boundary of a 
rectangle corresponding to a theoretical $100 \%$ release (Khan 1975).

Mathematical modeling of atrazine release The semiempirical Korsmeyer-Peppas model (Eq. 1) was applied to the herbicide release curves for the PCL NC prepared using different concentrations of chitosan, in order to elucidate the type of release mechanism (Siepmann and Siepmann 2008; Siepmann and Peppas 2001; Costa et al. 2001; Korsmeyer et al. 1983).

$\frac{M_{\mathrm{t}}}{M_{\infty}}=K t^{n}+b$

In Eq. $1, M_{\mathrm{t}} / M_{\infty}$ is the fraction of herbicide released at time $t, K$ is the release kinetic constant, which incorporates structural and geometric aspects of the process, and $n$ is the release exponent, which, according to its numerical value, characterizes the mechanism of release of the herbicide. This equation is generally used to interpret and describe the release of compounds when the predominant mechanism is not well known, or when it results from the combination of two apparently independent processes. One is due to herbicide transport obeying Fick's laws (Fickian transport), and the other (Case II transport) is a consequence of the swelling and relaxation of the polymeric matrix (dynamic expansion), involving a transition from a semi-rigid state to a more flexible condition. Korsmeyer et al. (1983) described the values of the release exponent as follows: $n<0.43$ indicates that the release mechanism is by pure diffusion (classical Fickian diffusion); $n>0.85$ reflects release modified by Case II transport, with swelling of the polymer (relaxation of the polymeric matrix); $0.43<n<0.85$ indicates anomalous transport, involving a combination of the mechanisms of diffusion and Case II transport.

\section{Results and discussion}

In earlier work (Grillo et al. 2012), PCL NC containing triazine herbicides (ATZ, ametryn, and simazine) were developed, achieving encapsulation efficiencies exceeding $84 \%$. The NC modified the release profiles of the herbicides, reduced the toxicity of the compounds, and remained stable over 270 days. Here, the PCL NC that had been prepared previously by Grillo et al. (2012) were coated with chitosan polymer.

The encapsulation efficiencies of the different formulations of PCL NC coated with chitosan (NC:PCL/CS) were compared (Fig. 1). The uncoated NC showed the highest encapsulation efficiency $(86.7 \pm 2.3 \%)$, followed by the $\mathrm{NC}$ prepared using the highest concentration of chitosan $(72.8 \pm 2.4 \%)$. The remaining formulations (prepared with $8,10,20$, and $30 \mu \mathrm{g} / \mathrm{mL}$ of chitosan) showed efficiencies close to $65 \%$. The greater encapsulation efficiency for the uncoated NC was probably due to the presence of ATZ adsorbed on the walls and surfaces of the NC. During the coating process, the herbicide was substituted by the chitosan polymer, resulting in the release of ATZ molecules into the solution and consequently diminishing the encapsulation efficiency. Nonetheless, even with these differences in encapsulation, all of the formulations presented a good interaction with the model molecule (ATZ), which could be explained by the interaction between ATZ, which is hydrophobic, and the oily core of the NC. Another factor that should be taken into consideration is that although the



Fig. 1 Encapsulation efficiencies of atrazine in PCL nanocapsules coated using different quantities of chitosan $(0,8,10,20,30$, and $40 \mu \mathrm{g} / \mathrm{mL})$. The experiments were performed in triplicate $(n=3)$ at $25^{\circ} \mathrm{C}$

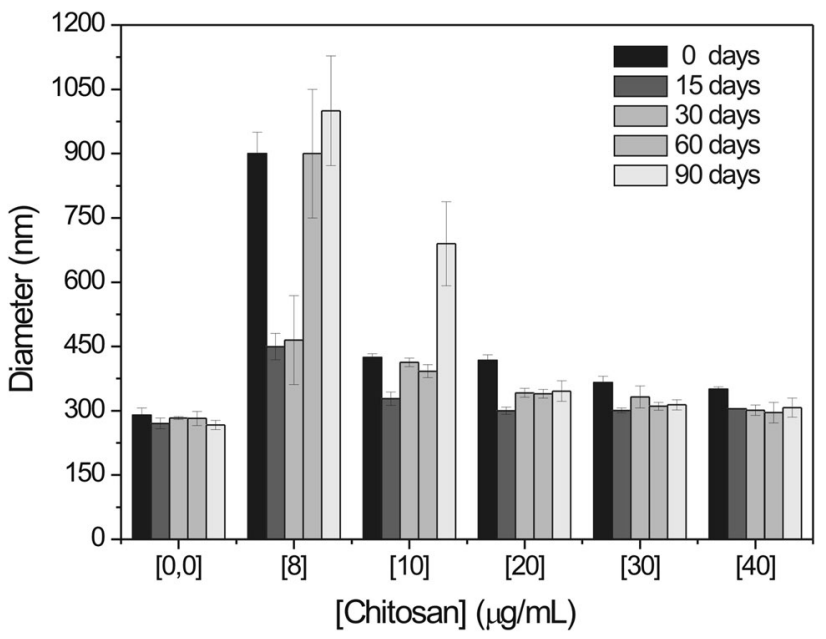

Fig. 2 Average size distribution of PCL nanocapsules coated using different concentrations of chitosan $(0,8,10,20,30$, and $40 \mu \mathrm{g} / \mathrm{mL})$ and containing atrazine, as a function of time $(0,15,30,60$, and 90 days). The experiments were performed in triplicate $(n=3)$ at $25^{\circ} \mathrm{C}$ 
Fig. 3 Size distributions of PCL nanocapsules coated with chitosan and containing atrazine: a nanocapsules prepared using $8 \mu \mathrm{g} / \mathrm{mL}$ of chitosan, on day 0 ;

b nanocapsules prepared using $40 \mu \mathrm{g} / \mathrm{mL}$ of chitosan, on day 0 ; c photographs of the formulations of nanocapsules coated with chitosan and containing atrazine, after a storage period of 90 days. The experiments were performed at $25{ }^{\circ} \mathrm{C}$
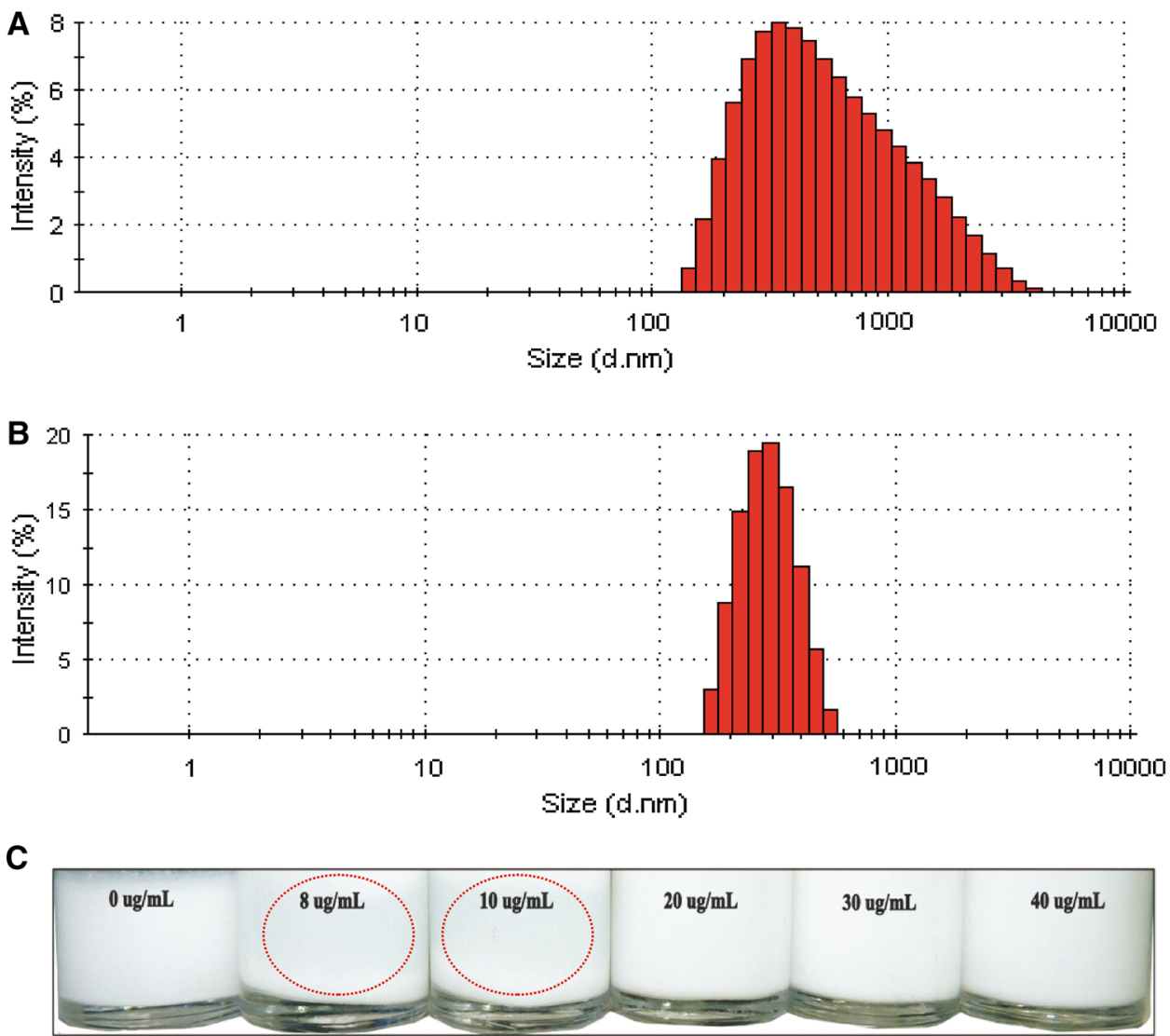

coated NC presented lower association efficiency, they should be able to provide better interaction with the longchain hydrocarbons, alcohols, acids, and triterpenes found in waxy plant cuticles (Baker 1982). This should improve the efficiency of absorption of the herbicide by the leaf, since the greater the retention of the active principle on the leaf surface, the greater its absorption and biological activity (Taylor 2011; Grangeot et al. 2006; Pathan et al. 2007).

The stability of the suspensions of NC:PCL/CS containing ATZ was evaluated using several different physicochemical parameters. Particle size (hydrodynamic diameter), polydispersion index, zeta potential, and $\mathrm{pH}$ were measured as a function of time (after $0,15,30,60$, and 90 days), during which all the samples were stored at $25{ }^{\circ} \mathrm{C}$ in flasks protected from light.

The effect of different concentrations of chitosan on the hydrodynamic diameter of the $\mathrm{NC}$ is shown in Fig. 2. There was an increase in particle size for all the formulations containing the cationic polymer, which provides evidence for the formation of a coating. At the beginning of the period ( 0 days), the size of the $\mathrm{NC}$ prepared using 8 and $10 \mu \mathrm{g} / \mathrm{mL}$ of chitosan was greater than that reported in the literature for these types of nanoparticles (Mora-Huertas et al. 2010). The NC containing $8 \mu \mathrm{g} / \mathrm{mL}$ of chitosan showed the greatest size changes over time, which could

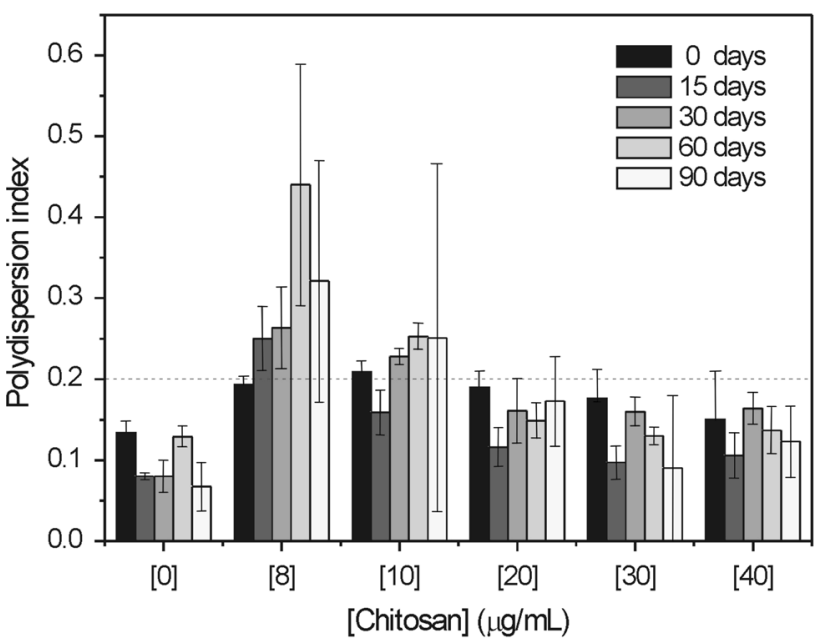

Fig. 4 Polydispersion index values for the PCL formulations prepared using different concentrations of chitosan $(0,8,10,20,30$, and $40 \mu \mathrm{g} / \mathrm{mL})$ and containing atrazine, as a function of time $(0,15$, 30,60 , and 90 days). The experiments were performed in triplicate $(n=3)$ at $25^{\circ} \mathrm{C}$

have been due to sudden shifts in the dynamics of the colloidal system, with electrostatic interactions initially causing particle agglomeration, after which (at between 15 and 30 days) the system became more organized, followed by a further loss of stability after 30 days. 
For the formulations of $\mathrm{NC}$ without chitosan $(0 \mu \mathrm{g} / \mathrm{mL})$, and the formulations containing 20,30 and $40 \mu \mathrm{g} / \mathrm{mL}$ of chitosan, the average size of the NC was stable over 90 days, and there was no evidence of aggregate formation. However, in the case of the formulations prepared with smaller quantities of chitosan ( 8 and $10 \mu \mathrm{g} / \mathrm{mL}$ ), the size of

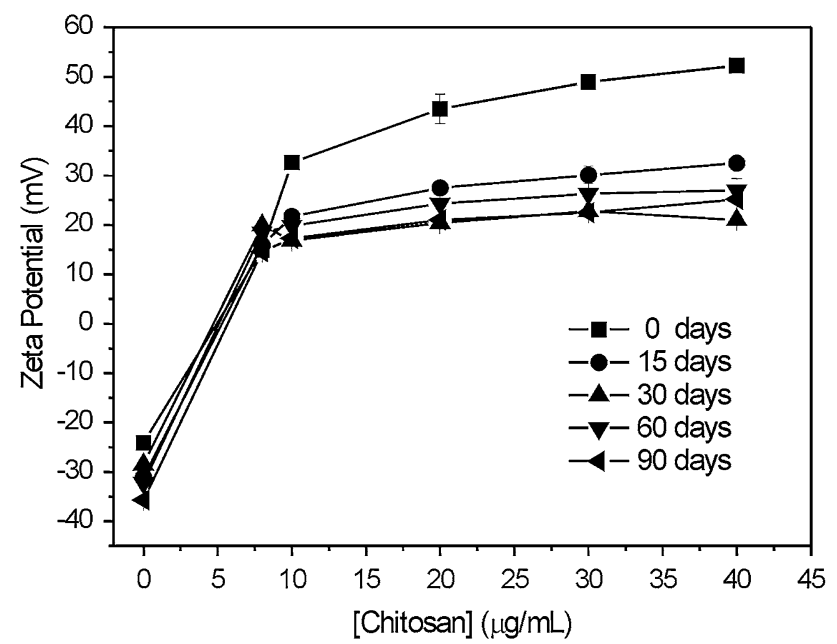

Fig. 5 Effect of increased concentrations of chitosan on the zeta potential values of PCL nanocapsules containing atrazine, as a function of time $(0,15,30,60$, and 90 days). The experiments were performed in triplicate $(n=3)$ at $25{ }^{\circ} \mathrm{C}$ the NC increased with time, and there were changes in the size distribution, as shown in Fig. 3a. The systems that used smaller concentrations of chitosan also exhibited greater variability and higher experimental errors, indicative of colloidal instability. On the other hand, the formulations containing higher concentrations of chitosan (20,

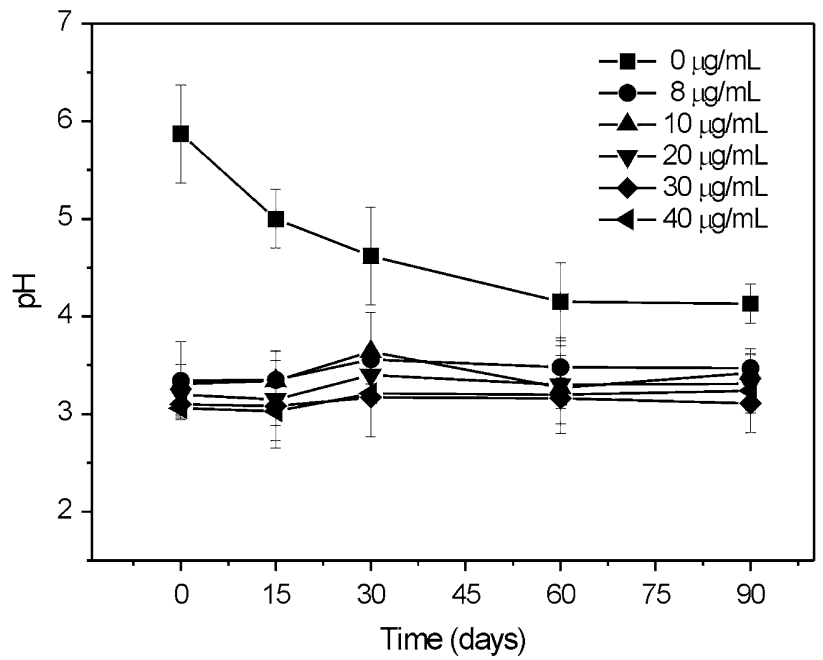

Fig. 6 Investigation of the $\mathrm{pH}$ of the formulations of PCL nanocapsules and PCL nanocapsules coated with chitosan, as a function of time $(0,15,30,60$, and 90 days). The experiments were performed in triplicate $(n=3)$ at $25{ }^{\circ} \mathrm{C}$

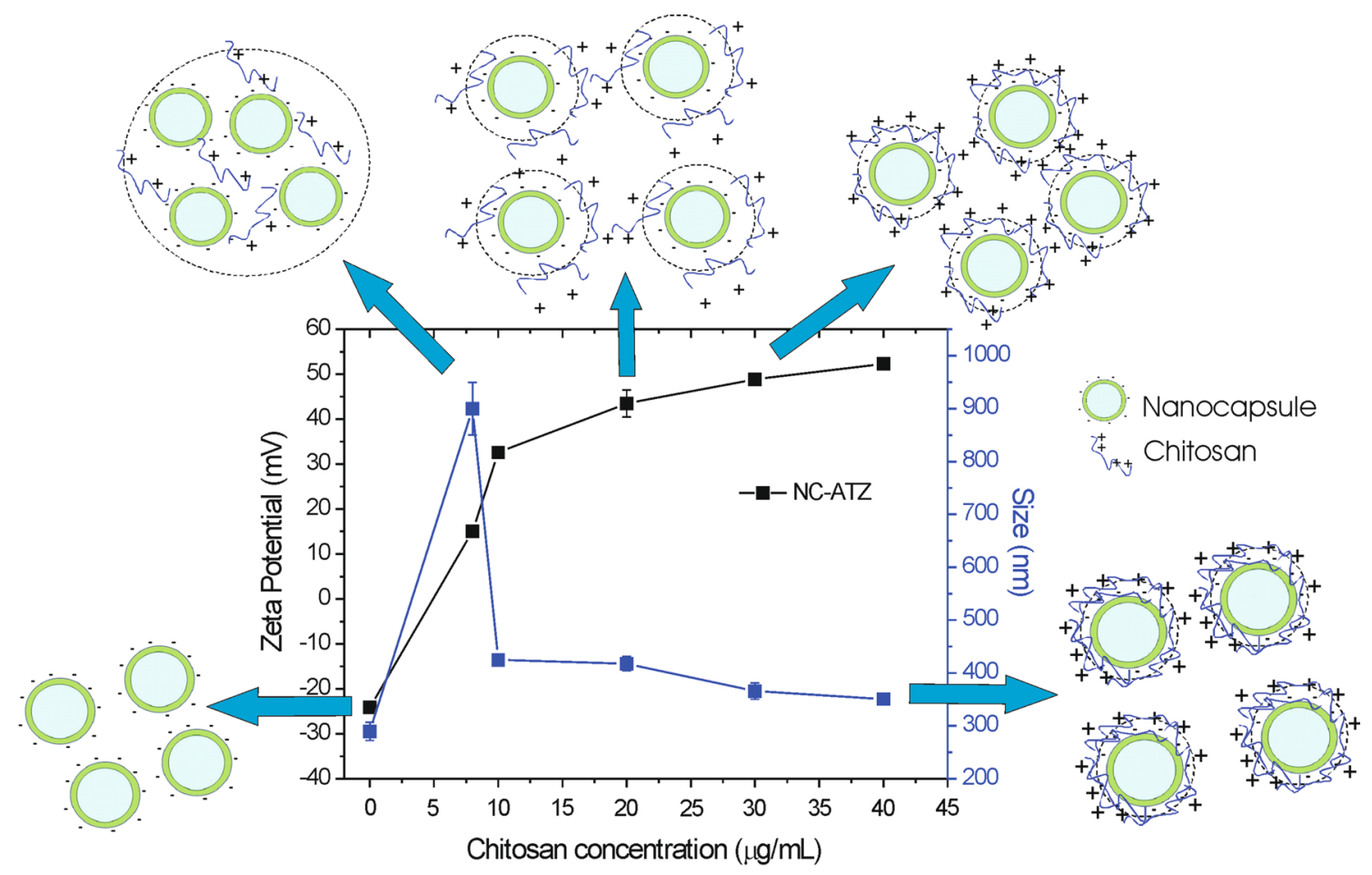

Fig. 7 Representation of the effect of increased chitosan concentrations on nanocapsule size and zeta potential, together with a schematic explanation of possible interactions between the positively charged groups of the chitosan polymer and the negative charges of the surface of the PCL nanocapsules containing atrazine 
30 , and $40 \mu \mathrm{g} / \mathrm{mL}$ ) presented particle sizes close to those reported in the literature $(200-500 \mathrm{~nm})$ and a single size distribution (Fig. 3b).

Evaluation of the size of the coated NC provided important information concerning the interaction of chitosan with the polymeric wall. The experiments demonstrated that low concentrations of chitosan increased the size of the NC and decreased the temporal stability, probably due to the formation of aggregates. When the concentration of chitosan was increased, the NC were more thickly coated, more consistent in size, and remained more stable. Figure $3 \mathrm{c}$ shows the behavior of the formulations after 90 days of storage at $25{ }^{\circ} \mathrm{C}$, revealing the presence of aggregates in the formulations prepared with smaller concentrations of chitosan (8 and $10 \mu \mathrm{g} / \mathrm{mL}$ ).

Most of the formulations presented a polydispersion index below 0.2 , which is associated with a monodisperse distribution and good homogeneity (Mora-Huertas et al. 2010). Only the NC formulations prepared using 8 and $10 \mu \mathrm{g} / \mathrm{mL}$ of chitosan showed polydispersion indices above 0.3 , indicative of systems with distributions of differently sized particles, as illustrated in Fig. 4.

The zeta potential obtained for the uncoated NC containing ATZ was approximately $-40 \mathrm{mV}$, due to the presence of carboxylic groups in the PCL polymer as well as other components of the formulation, including the surfactant located at the polymer/water interface (Fig. 5). The potential was shifted to positive values for the formulations containing chitosan at different concentrations (Fig. 5), indicating that there was an increase in positively charged amino groups of the chitosan molecules, proving that the nanoparticles had been successfully coated. Similar results have been reported in the literature for ocular and buccal NC (Calvo et al. 1997; Mazzarino et al. 2012).

The charges measured on day 0 increased proportionately to the amount of chitosan used, and this trend was maintained over time. The zeta potential values of all the samples diminished with time, which could be explained by hydrolysis of the PCL polymer and consequent release of carboxylic groups.

The $\mathrm{pH}$ of the formulations without chitosan decreased with time (Fig. 6), evidencing polymer degradation, as found previously (Guterres et al. 2010; Schaffazick et al. 2003). The $\mathrm{pH}$ of the coated NC formulations was close to 3 and showed no significant change over 90 days (Fig. 6), as a result of which any degradation of the PCL polymer was not observed. The high $\mathrm{H}^{+}$concentration found for the coated formulations was due to the acetic acid solution used to dissolve the chitosan in the initial preparation process. An acid $\mathrm{pH}$ is required in order to generate protonated groups on the chitosan and facilitate electrostatic interaction with the PCL polymer.
The stability data were used to characterize the behavior of the polymeric $\mathrm{NC}$ by construction of a representation relating NC size to the zeta potential (Fig. 7). These data clearly indicated that the adsorption process was mediated by electrostatic interactions (Calvo et al. 1997). The zeta potential increased as the formulations received increasing quantities of chitosan, while the NC size increased when low concentrations of chitosan were used in the formulation and decreased when higher concentrations were used (Fig. 7). A possible explanation is that when the NC began to be coated by the chitosan, they shifted from a low zeta potential $(-30 \mathrm{mV})$, at which they possessed substantial electrostatic repulsion, to a positive zeta potential, at which there was less electrostatic repulsion and greater particle agglomeration. However, further studies will be needed in order to understand the nature of this agglomeration, which
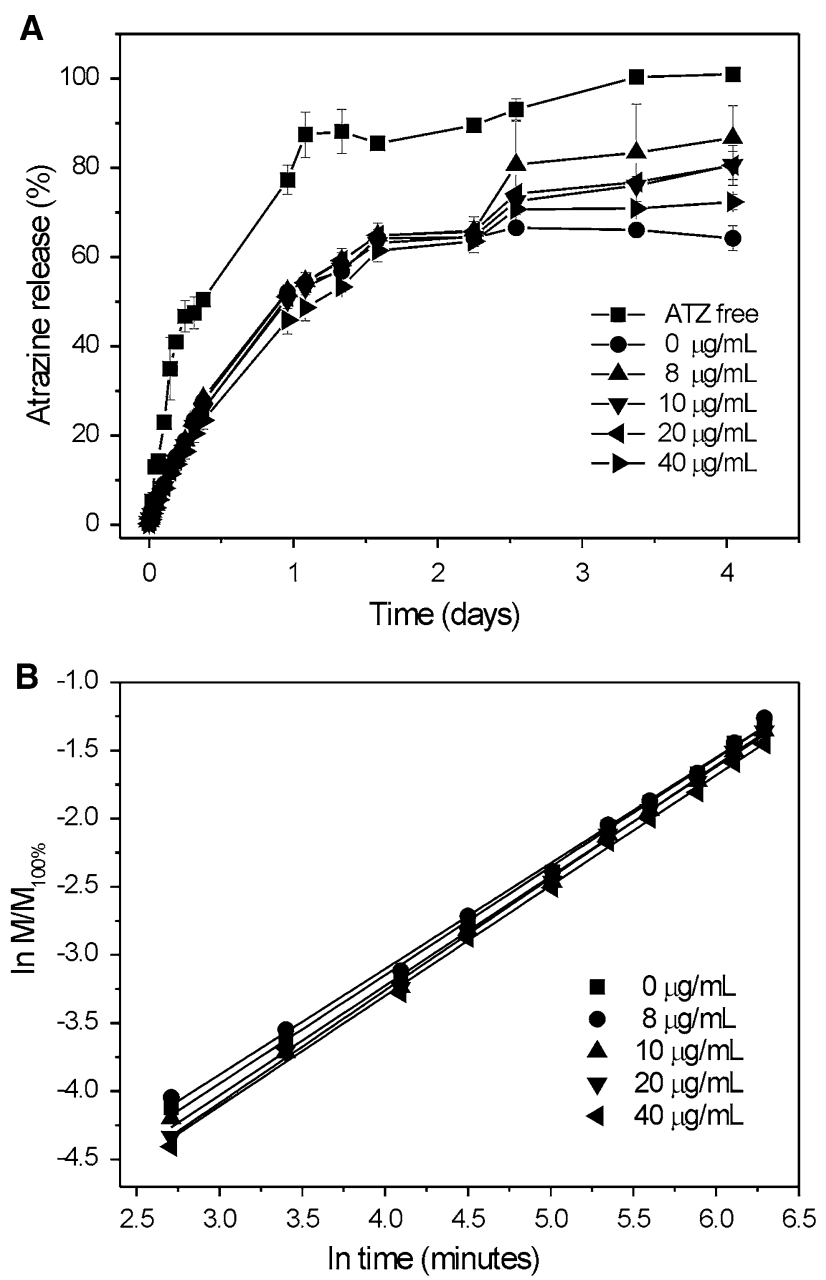

Fig. 8 Kinetic release curves of atrazine associated with coated and uncoated PCL nanocapsules. a In vitro release kinetics, at $25^{\circ} \mathrm{C}$, for PCL nanocapsules coated using different concentrations of chitosan, measured in triplicate $(n=3)$. b Results obtained following application of the Korsmeyer-Peppas mathematical model to the atrazine release curves obtained for the coated and uncoated PCL nanocapsules 
Table 1 Atrazine release efficiencies for formulations of PCL nanocapsules coated with chitosan

\begin{tabular}{ll}
\hline NC:PCL/CS [chitosan] $(\mu \mathrm{g} / \mathrm{mL})$ & Release efficiency (RE) \\
\hline 0 & $56.2 \% \pm 1.2$ \\
8 & $63.9 \% \pm 0.9$ \\
10 & $59.8 \% \pm 0.8$ \\
20 & $60.0 \% \pm 1.1$ \\
40 & $56.5 \% \pm 0.5$ \\
\hline
\end{tabular}

could be due to Ostwald ripening or a combination of flocculation and coalescence processes. When higher concentrations of chitosan ( 30 and $40 \mu \mathrm{g} / \mathrm{mL}$ ) were used, the zeta potential increased $(>45 \mathrm{mV})$, resulting in repulsion between the $\mathrm{NC}$ and creation of a more stable system with size, polydispersion index, and zeta potential values that were characteristic of stable colloids (Calvo et al. 1997). Similar results have been reported in the literature for nanoparticles of $\mathrm{TiO}_{2}$ submitted to different $\mathrm{pH}$ conditions. Measurements of particle size and zeta potential revealed that when the $\mathrm{pH}$ approached the isoelectric point, the repulsive force was weakened due to the low surface charge, and the hydrodynamic size increased to beyond the measurable range. Under these conditions, large flocs were formed, which rapidly settled out of the solution due to gravitational forces (Jiang et al. 2009).

The formulations containing higher quantities of chitosan (30 and $40 \mu \mathrm{g} / \mathrm{mL}$ ) therefore provided better coating of the PCL NC, suggesting that these formulations should be the most promising in future investigations of the use of these systems against target organisms.

Release kinetics experiments were performed to determine how chitosan might affect the release profile of the active principle, and whether any change occurred in the mechanism of release of ATZ from the coated PCL NC. Figure 8a shows the release profiles of free ATZ (ATZ dissolved in water), ATZ NC without chitosan $(0 \mu \mathrm{g} / \mathrm{mL})$, and ATZ from the coated $\mathrm{NC}$, as a function of time and the different chitosan concentrations employed. The release profiles of all the formulations were slower, compared to free ATZ. The formulations of uncoated $\mathrm{NC}(0 \mu \mathrm{g} / \mathrm{mL})$ and $\mathrm{NC}$ coated with the greatest quantity of chitosan showed similar profiles, which were slower compared to the profiles obtained for the formulations prepared using the other concentrations of CS.

These findings can be explained by the differences in the encapsulation efficiencies (illustrated in Fig. 1). The formulation prepared using the lowest concentration of chitosan $(8 \mu \mathrm{g} / \mathrm{mL})$ showed the fastest release profile of all the formulations, due to the diminished encapsulation efficiency caused by the removal of ATZ from the surface of the NC. It can be concluded that low concentrations of chitosan destabilized the NC system and accelerated the release of ATZ; the release was faster due to removal of the ATZ molecules adhering to the surface of the coated NC.

The release efficiency describes the quantity (as a percentage) of active principal released in the system and is calculated by comparing the area of the theoretical rectangle corresponding to $100 \%$ release with the area under the experimentally determined release profile curve (Costa et al. 2001). From the calculated RE values (Table 1), it can be seen that the formulations prepared using 0 and $40 \mu \mathrm{g} / \mathrm{mL}$ of chitosan presented similar release profiles, with both systems retarding the release of ATZ by around $43 \%$ over the trial period. RE values between 75 and $87 \%$ have been reported in the literature for polymeric microparticles containing ametryn (Grillo et al. 2011), which are of similar order as the values obtained here for the polymeric NC.

The mechanism of release of ATZ from the different coated NC was investigated using the Korsmeyer-Peppas theoretical model (Costa et al. 2001). Linear regressions were applied to the release curves in order to determine the correlation coefficients $(r)$ and the release constants $(k)$. Table 2 gives the results obtained using the data illustrated in Fig. 8b. Application of the Korsmeyer-Peppas mathematical treatment resulted in values of the constant $(k)$ that varied between $1.4 \times 10^{-4}$ and $2.0 \times 10^{-4} / \mathrm{min}$, with higher values being indicative of faster release of the herbicide. The values of the release exponent $(n)$ varied between 0.77 and 0.82 , indicating that release of the herbicide from the interior of the coated NC occurred due to a combination of non-Fickian diffusion and relaxation of the polymeric chains. In comparison, the release exponent values obtained for uncoated PCL nanoparticles containing
Table 2 Values of the release constant $(k)$, release exponent $(n)$, and correlation coefficient ( $r$ ) obtained for the best fits of the release kinetics curves for atrazine associated with the PCL nanocapsules coated with chitosan

\begin{tabular}{llll}
\hline $\begin{array}{l}\text { NC:PCL/CS [chitosan] } \\
(\mu \mathrm{g} / \mathrm{mL})\end{array}$ & $\begin{array}{l}\text { Release constant } \\
(k)\left(\mathrm{min}^{-1}\right)\end{array}$ & $\begin{array}{l}\text { Release exponent } \\
(n)\end{array}$ & $\begin{array}{l}\text { Correlation coefficient } \\
(r)\end{array}$ \\
\hline 0 & $1.7 \times 10^{-4}$ & 0.89 & 0.997 \\
8 & $2.0 \times 10^{-4}$ & 0.77 & 0.998 \\
10 & $1.5 \times 10^{-4}$ & 0.80 & 0.997 \\
20 & $1.4 \times 10^{-4}$ & 0.82 & 0.998 \\
40 & $1.4 \times 10^{-4}$ & 0.81 & 0.997 \\
\hline
\end{tabular}


ATZ (Grillo et al. 2012) were indicative of a mechanism that only involved relaxation of the polymeric chains. Silva et al. (2010), (2011) prepared alginate/chitosan nanoparticles containing the herbicides paraquat and clomazone, and obtained release exponent values between 0.67 and 0.83 , showing that the particles containing chitosan released the herbicide according to the same mechanism proposed in the present work.

\section{Conclusion}

Formulations of NC coated with chitosan and containing ATZ showed encapsulation efficiencies exceeding $65 \%$, with different values obtained depending on the concentration of chitosan used. The release profile of atrazine was altered by the presence of chitosan on the surface of the $\mathrm{NC}$, compared to uncoated NC. The mechanism of release from the coated NC involved non-Fickian diffusion and relaxation of the polymeric chains, which could be explained by alterations in the dynamics of the electrostatic interactions between the polymeric chains of chitosan and the PCL NC. The size and polydispersion of the particles were greater for NC coated using a low concentration of chitosan, and smaller for formulations prepared with higher concentrations, resulting in differences in particle aggregation. The zeta potential of the formulations was shifted from negative to positive with the addition of chitosan, indicative of changes in the conformation of the polymeric wall. The findings open new perspectives for modified herbicide release systems, since it has been shown that changes in the surface charge of NC can improve their adhesion to the target substrate. This should assist the development of more efficient systems for the control of weeds in agriculture.

Acknowledgments The authors thank FAPESP, CNPq, and FUNDUNESP for financial support.

\section{References}

Baker EA (1982) Chemistry and morphology of plant epicuticular waxes. In: Cutler DJ, Alvin KL, Price AL (eds) The plant cuticle. Academic Press, London, pp 139-165

Bilensoy E, Sarisozen C, Esendagli G, Dogan AL, Aktas Y, Sen M, Mungan NA (2009) Intravesical cationic nanoparticles of chitosan and polycaprolactone for the delivery of Mitomycin C to bladder tumors. Int J Pharm 371(1-2):170-176. doi:10.1016/j. ijpharm.2008.12.015

Breckenridge CB, Eldridge JC, Stevens JT, Simpkins JW (2010) Symmetrical triazine herbicides: a review of regulatory toxicity endpoints. In: Krieger RI, Hayes WJ (eds) Handbook of pesticide toxicology, vol 2. 3rd edn. Elsevier/Academic Press, Boston, pp 1711-1723

Calvo P, VilaJato JL, Alonso MJ (1997) Evaluation of cationic polymer-coated nanocapsules as ocular drug carriers. Int J Pharm 153(1):41-50. doi:10.1016/S0378-5173(97)00083-5
Chakravarthi SS, Robinson DH (2011) Enhanced cellular association of paclitaxel delivered in chitosan-PLGA particles. Int $\mathrm{J}$ Pharm 409(1-2):111-120. doi:10.1016/j.ijpharm.2011.02.034

Clemente-Napimoga JT, Moreira JA, Grillo R, de Melo NFS, Fraceto LF, Napimoga MH (2012) 15d-PGJ2-loaded in nanocapsules enhance the antinociceptive properties into rat temporomandibular hypernociception. Life Sci 90(23-24):944-949. doi:10. 1016/j.lfs.2012.04.035

Costa P, Manuel J, Lobo S (2001) Modeling and comparison of dissolution profiles. Eur J Pharm Sci 13(2):123-133. doi:10. 1016/S0928-0987(01)00095-1

Dash TK, Konkimalla VB (2012) Poly-epsilon-caprolactone based formulations for drug delivery and tissue engineering: a review. J Control Release 158(1):15-33. doi:10.1016/j.jconrel.2011.09. 064

de Melo NFS, Grillo R, Guilherme VA, de Araujo DR, de Paula E, Rosa AH, Fraceto LF (2011) Poly(lactide-co-glycolide) nanocapsules containing benzocaine: influence of the composition of the oily nucleus on physico-chemical properties and anesthetic activity. Pharm Res 28(8):1984-1994. doi:10.1007/s11095-0110425-6

Fessi H, Puisieux F, Devissaguet JP, Ammoury N, Benita S (1989) Nanocapsule formation by interfacial polymer deposition following solvent displacement. Int J Pharm 55(1):R1-R4. doi:10. 1016/0378-5173(89)90281-0

Garcia-Fuentes M, Alonso MJ (2012) Chitosan-based drug nanocarriers: where do we stand? J Control Release 161(2):496-504. doi:10.1016/j.jconrel.2012.03.017

Govender T, Riley T, Ehtezazi T, Garnett MC, Stolnik S, Illum L, Davis SS (2000) Defining the drug incorporation properties of PLA-PEG nanoparticles. Int J Pharm 199(1):95-110. doi:10. 1016/S0378-5173(00)00375-6

Grangeot M, Chauvel B, Gauvrit C (2006) Spray retention, foliar uptake and translocation of glufosinate and glyphosate in Ambrosia artemisiifolia. Weed Res 46(2):152-162. doi:10. 1111/j.1365-3180.2006.00495.x

Gref R, Quellec P, Sanchez A, Calvo P, Dellacherie E, Alonso MJ (2001) Development and characterization of CyA-loaded poly(lactic acid)poly(ethylene glycol)PEG micro- and nanoparticles. Comparison with conventional PLA particulate carriers. European Journal of Pharmaceutics and Biopharmaceutics 51(2):111-118. doi:10.1016/S0939-6411(00)00143-0

Grillo R, de Melo NFS, de Araujo DR, de Paula E, Rosa AH, Fraceto LF (2010a) Polymeric alginate nanoparticles containing the local anesthetic bupivacaine. J Drug Target 18(9):688-699. doi:10. 3109/10611861003649738

Grillo R, de Melo NFS, de Lima R, Lourenco RW, Rosa AH, Fraceto LF (2010b) Characterization of atrazine-loaded biodegradable poly(hydroxybutyrate-co-hydroxyvalerate) microspheres. J Polym Environ 18(1):26-32. doi:10.1007/s10924-009-0153-8

Grillo R, Pereira Ado E, de Melo NF, Porto RM, Feitosa LO, Tonello PS, Dias Filho NL, Rosa AH, Lima R, Fraceto LF (2011) Controlled release system for ametryn using polymer microspheres: preparation, characterization and release kinetics in water. J Hazard Mater 186(2-3):1645-1651. doi:10.1016/j. jhazmat.2010.12.044

Grillo R, dos Santos NZP, Maruyama CR, Rosa AH, de Lima R, Fraceto LF (2012) Poly(epsilon-caprolactone)nanocapsules as carrier systems for herbicides: physico-chemical characterization and genotoxicity evaluation. J Hazard Mater 231:1-9. doi:10. 1016/j.jhazmat.2012.06.019

Gupta NK, Tomar P, Sharma V, Dixit VK (2011) Development and characterization of chitosan coated poly-(varepsilon-caprolactone) nanoparticulate system for effective immunization against influenza. Vaccine 29(48):9026-9037. doi:10.1016/j.vaccine. 2011.09.033 
Guterres SS, Poletto FS, Colomé LM, Raffin RP, Pohlmann AR (2010) Polymeric nanocapsules for drug delivery an overview. In: Fanun M (ed) Colloids in drug delivery, vol 1. 3rd edn. CRC Press, pp 71-98

Hack B, Egger H, Uhlemann J, Henriet M, Wirth W, Vermeer AWP, Duff DG (2012) Advanced agrochemical formulations through encapsulation strategies? Chem Ing Tech 84(3):223-234. doi:10. $1002 /$ cite. 201100212

ICH (1996) Guidance for Industry-Q2B. Validation of analytical procedures. In: International conference on harmonization

Jiang JK, Oberdorster G, Biswas P (2009) Characterization of size, surface charge, and agglomeration state of nanoparticle dispersions for toxicological studies. J Nanopart Res 11(1):77-89. doi:10.1007/s11051-008-9446-4

Khan KA (1975) The concept of dissolution efficiency. J Pharm Pharmacol 27(1):48-49

Korsmeyer RW, Gurny R, Doelker E, Buri P, Peppas NA (1983) Mechanisms of solute release from porous hydrophilic polymers. Int J Pharm 15:25-35

Kumar P, Kumar P, Rajoria G, Narang RK (2012) Techniques for preparation of pharmaceutical coated nanoparticles: a comprehensive review. Am J Pharm Tech Res 3:121-135

Maynard AD (2011) Don't define nanomaterials. Nature 475:31

Mazzarino L, Travelet C, Ortega-Murillo S, Otsuka I, PignotPaintrand I, Lemos-Senna E, Borsali R (2012) Elaboration of chitosan-coated nanoparticles loaded with curcumin for mucoadhesive applications. J Colloid Interface Sci 370(1):58-66. doi:10.1016/j.jcis.2011.12.063

Moraes CM, de Matos AP, Grillo R, de Melo NF, de Paula E, Dias Filho NL, Rosa AH, Fraceto LF (2011) Screening of formulation variables for the preparation of poly(epsilon-caprolactone) nanocapsules containing the local anesthetic benzocaine. J Nanosci Nanotechnol 11(3):2450-2457

Mora-Huertas CE, Fessi H, Elaissari A (2010) Polymer-based nanocapsules for drug delivery. Int J Pharm 385(1-2):113-142. doi:10.1016/j.ijpharm.2009.10.018

Nagarwal RC, Kumar R, Pandit JK (2012) Chitosan coated sodium alginate-chitosan nanoparticles loaded with 5-FU for ocular delivery: in vitro characterization and in vivo study in rabbit eye. Eur J Pharm Sci 47(4):678-685. doi:10.1016/j.ejps.2012.08.008

Paavola A, Yliruusi J, Kajimoto Y, Kalso E, Wahlstrom T, Rosenberg $\mathrm{P}$ (1995) Controlled release of lidocaine from injectable gels and efficacy in rat sciatic nerve block. Pharm Res 12(12):1997-2002. doi:10.1023/A:1016264527738

Pathan AK, Kimberley MO, Forster WA, Steele KD, Gaskin RE (2007) Effects of retention and uptake on biological performance of glyphosate. In: Gaskyn RE (ed) Proceedings of the 8th International Symposium on Adjuvants for Agrochemicals. International Society for Agrochemical Adjuvants, USA

Prego C, Garcia M, Torres D, Alonso MJ (2005) Transmucosal macromolecular drug delivery. J Control Release 101(1-3):151162. doi:10.1016/j.jconrel.2004.07.030

Radad K, Al-Shraim M, Moldzio R, Rausch WD (2012) Recent advances in benefits and hazards of engineered nanoparticles. Environ Toxicol Pharmacol 34(3):661-672. doi:10.1016/j.etap. 2012.07.011

Sabir MI, Xu XX, Li L (2009) A review on biodegradable polymeric materials for bone tissue engineering applications. J Mater Sci 44(21):5713-5724. doi:10.1007/s10853-009-3770-7

Schaffazick SR, Guterres SSU, Freitas LD, Pohlmann AR (2003) Physicochemical characterization and stability of the polymeric nanoparticle systems for drug administration. Quim Nova 26(5):726-737

Siepmann J, Peppas NA (2001) Mathematical modeling of controlled drug delivery. Adv Drug Deliv Rev 48(2-3):137-138

Siepmann J, Siepmann F (2008) Mathematical modeling of drug delivery. Int J Pharm 364(2):328-343. doi:10.1016/j.ijpharm. 2008.09.004

Silva MD, Cocenza DS, de Melo NFS, Grillo R, Rosa AH, Fraceto LF (2010) Alginate nanoparticles as a controlled release system for clomazone herbicide. Quim Nova 33(9):1868-1873. doi:10. 1590/S0100-40422010000900009

Silva MD, Cocenza DS, Grillo R, de Melo NFS, Tonello PS, de Oliveira LC, Cassimiro DL, Rosa AH, Fraceto LF (2011) Paraquat-loaded alginate/chitosan nanoparticles: preparation, characterization and soil sorption studies. J Hazard Mater 190(1-3):366-374. doi:10.1016/j.jhazmat.2011.03.057

Silva MD, Cocenza DS, Rosa AH, Fraceto LF (2012) Effect of the association of clomazone in alginate/chitosan nanoparticles under soil sorption. Quim Nova 35(1):102-107

Taylor P (2011) The wetting of leaf surfaces. Curr Opin Colloid In 16(4):326-334. doi:10.1016/j.cocis.2010.12.003

Tobio M, Sanchez A, Vila A, Soriano I, Evora C, Vila-Jato JL, Alonso MJ (2000) The role of PEG on the stability in digestive fluids and in vivo fate of PEG-PLA nanoparticles following oral administration. Colloid Surf B 18(3-4):315-323. doi:10.1016/ S0927-7765(99)00157-5

Wu L, Zhang J, Watanabe W (2011) Physical and chemical stability of drug nanoparticles. Adv Drug Deliv Rev 63(6):456-469. doi:10.1016/j.addr.2011.02.001 